\title{
INFORMACIONES
}

\section{ACTIVIDADES DE LOS INVESTIGADORES DURANTE 1990}

La Doctora Beatriz de la Fuente dictó las siguientes conferencias: "La aproximación estética-psicológica de Westheim al arte antiguo de México", en el Museo del Palacio de Bellas Artes, mayo 15; "Homenaje a Manuel Toussaint" en El Colegio Nacional, julio 26; "Escultura prehispánica" en la Academia de Artes, septiembre 25; "El arte olmeca" en la Universidad Iberoamericana, octubre 24; coordinó el curso de diez conferencias sobre "La pintura mural prehispánica" del 26 de noviembre al 7 de diciembre en El Colegio Nacional, allí dictó la conferencia, "La figura humana en la pintura mural", el 27 de noviembre.

Participó y asistió a los congresos: Maya Civilization of Cycle 6 and 7: Classic Maya in the Preclassic, con la ponencia "Olmec sculpture as apprecciated from the History of Art", en el departamento de Antropología de Berkeley, California, el 21 de agosto. Cuartas Jomadas de Historiadores Americanistas: Religión y Cosmos en el Mundo Prehispánico, con la ponencia "Homocentrismo en el arte olmeca", en Santa Fe de Granada, España, el 16 de octubre. Asistió, en su calidad de vicepresidente, al Coloquio Anual del Comité International d'Histoire de l'Art, en Bolonia, Italia, del 11 al 14 de octubre.

Docencia y dirección de tesis: dictó dos seminarios semestrales en la División de Estudios de Posgrado de la Facultad de Filosofía y Letras, con el tema "Figuras prehispánicas con posible significación ígnea". Dirige dos tesis a nivel de maestría y una de doctorado.

Publicaciones: "George Kubler" y "Plantas alucinógenas en lápidas de Tula" en World Art: Themes of University in Diversity. Acts of the XXVI International Congress of the History of Art. The Pennsylvania State University Press, 1989, pp. 25 y 26, 600-609, respectivamente. "Octavio Paz y el mundo prehispánico" en Octavio Paz Los privilegios de la vista. Centro Cultural de Arte Contemporáneo, México, 1990, pp. 63-71. "Retorno al pasado tolteca" en Artes de México. Nueva época, Arte del Templo Mayor, número 7, Primavera, 1990, pp. 36-54. "La escultura maya clásica" en Los Mayas. Esplendor de una civilización. Sociedad Estatal Quinto Centenario, Turner Libros, Madrid, 1990, pp. 73-85. "An- 
cient Mexican Art: Diversity in Unity" en Mexico: Splendors of Thirty Centuries. The Metropolitan Museum of Art, New York, 1990, pp. 41-51.

Dos reseñas de libros publicadas en Anales del IIE, vol. XV, no. 60.

Distinciones y nombramientos: en enero 8 se le nombró Miembro del Consejo Consultivo de Ciencias de la Presidencia de la República; en mayo 8 se le otorgó la medalla de 25 años como profesora e investigadora de la UNAM; en octubre fue jurado de los premios Universidad Nacional y Distinción Universidad Nacional.

Otras actividades: Entrevistas durante febrero y marzo, para ser transmitidas en los canales 9 y 11 de televisión, sobre el Premio Nacional de Ciencias y Artes en el campo de Historia, Ciencias Sociales y Filosofía, que le fue otorgado en diciembre de 1989. Entrevista para el diario japonés Yomiuri, publicada los días 24 y 25 de enero.

Invitada el 25 y 26 de junio a Dallas, como asesora de la exposición de Arte Olmeca que se presentará en 1992-1993, en el Dallas Museum of Art y la National Gallery of Washington.

Como directora de la Colección de Arte de la UNAM, supervisó el no. 44, Artificios del Barroco. México y Puebla en el siglo XVII, por Martha Fernández.

Xavier Moyssén coordinó con varios miembros del Instituto y del Museo Nacional de Arte, la exposición homenaje a don Manuel Toussaint con motivo del primer centenario de su nacimiento. También coordinó la publicación del catálogo de la exposición.

Intervino con el señor Danilo Ongay Muza en la preparación de los originales para la edición de los libros de don Manuel Toussaint Arte Colonial en México y Pintura Colonial en México.

Durante el año dictó varias conferencias sobre arte contemporáneo en México. Participó con un trabajo para la Mesa Redonda que organizó en homenaje al arquitecto Augusto $\mathrm{H}$. Álvarez, la Academia Mexicana de Arquitectura.

Gustavo Curiel participó en el libro: Catálogo. Obras maestras del Arte Colonial. Exposición Homenaje a Manuel Toussaint 1890-1990. Tuvo a su cargo la elaboración de seis comentarios de piezas de la muestra. 
Preparó la edición de los textos que conforman el libro: Imaginería virreinal. Memorias de un Seminario. Preparó una advertencia y las conclusiones, así como también un artículo. México, UNAM, Instituto de Investigaciones Estéticas/INAH, 1990.

Entregó para su publicación en las Memorias del Segundo Congreso de Historia Regional Comparada el artículo "Ajuares domésticos en el Parral de los siglos xviI y XviII”, México, Universidad Autónoma de Ciudad Juárez, 1991.

Fue entregado para su publicación en el Tomo IV del estudio: Juan Correa. Su vida y su obra el artículo: "Las pinturas de la Virgen de los Dolores y la Piedad en la obra de Juan Correa", México, UNAM, Instituto de Investigaciones Estéticas, en prensa.

Participó con la ponencia: "Tránsito de obras suntuarias a la Nueva España. Reflexiones sobre el comercio transmarítimo" en el Primer Simposio Internacional "España y Nueva España: sus acciones transmarítimas", noviembre 1990. El texto se recogerá en las Memorias del Simposio. México, Universidad Iberoamericana.

Entregó para la revista Memoria, del Museo Nacional de Arte tres comentarios a obras expuestas en la muestra homenaje al fundador del Instituto de Investigaciones Estéticas.

Asesoría a la Secretaría de Hacienda y Crédito Público a través de la Dirección General de Control del Acervo Patrimonial.

Formó parte del Comité Editorial del Instituto.

Impartió el Seminario de Arte Colonial, nivel licenciatura, en la Facultad de Filosofía y Letras de la UNAM.

El arquitecto Manuel González Galván dictó las siguientes conferencias:

"Identidad Nacional en el arte". Fúndice, enero 17.

"Presencias potosinas en el arte virreinal". Consejo Estatal para la Cuitura y las Ârtes, Casa de la Cultura, San Luis Potosí, enero 26.

"Catedral de Morelia, proporción y símbolo", IV Reunión ACBI, (Asociación de Conservadores de Bienes Inmuebles). León, Gto., febrero 17.

"Sobre iconología". ACBI. Morelia, Mich., mayo 19.

"El barroco salomónico". Fúndice, julio 25.

"Singularidades michoacanas de la iconografía". ACBI, Morelia, Mich., agosto 18. 
Asistió a los congresos y coloquios a continuación enumerados:

Primera Asamblea General de la Comisión Nacional para la preservación del Patrimonio Cultural de la Nación, de la cual es miembro, en Tajín, Ver., del 19 al 21 de abril.

Asistió a las Jornadas Alarconianas en Taxco, Gro., por invitación del Gobierno del Edo. de Guerrero, mayo 26 y 27.

Asistencia a la XI Reunión Nacional del INBA. Ixtapan de la Sal, Méx., del 28 al 30 de junio, con la ponencia, "La promoción cultural y la defensa del Patrimonio Artístico".

Asistió al Coloquio en Homenaje a Manuel Toussaint, en Tlaxcala, Tlax., del 1 al 3 de agosto, con la ponencia dictada en vivo sobre "Iconogesis en el Santuario de Ocotlán".

Asistió al Seminario sobre Santa Prisca de Taxco. Una revisión histórica y estética. Los días 12 y 13 de octubre, con la ponencia, "Ordenada voz iconológica".

Asistió al coloquio anual del icomos Mexicano, en la ciudad de México, del 7 al 9 de noviembre.

Impartió los cursos de "Iconología" en Oaxaca, Oax., con visitas a monumentos, del 3 al 7 de julio. y de "Arte Barroco", a nivel posgrado, en la Facultad de Arquitectura, UNAM, del 21 al 28 de septiembre.

Otorgó las siguientes entrevistas:

"Problemas para la preservación del patrimonio cultural". Periódico La Voz de Michoacán, enero 28.

"Ser o no ser". Crítica de arquitectura, revista Obras, núm. 207, Vol. XVIII, marzo 1.

"El Colegio de Vizcaínas". T.V. Canal 7, IMEvisión, marzo 14.

"El Centro Histórico de la Cd. de México". T.V. Canal 7, IMEvisión, abril 1.

Fue jurado en la selección de pintores a exponer en la Galería del Instituto Mexicano de Relaciones Culturales, enero 31.

Asesoró en varias reuniones del Consejo de Monumentos Históricos del INAH.

En otras actividades. El día 27 de octubre se inauguró el Mausoleo de Don Vasco de Quiroga, a donde fueron trasladados sus restos ese mismo día, después de anticipadas y solemnes ceremonias cívicas y religiosas. Dicho Mausoleo, que se ubica en el interior de una de las capillas de la Basilica de Nuestra Señora de la Salud en Pátzcuaro, Mich., fue diseñado y vigiló su ejecución el mismo arquitecto González Galván. 
La doctora Elisa García Barragán, como Directora del Instituto de Investigaciones Estéticas, organizó y coordinó las siguientes actividades: logró la participación del Consejo Nacional para la Cultura y las Artes, el Instituto Nacional de Bellas Artes y el Departamento del Distrito Federal para la realización de una serie de homenajes a Manuel Toussaint fundador del Instituto de Investigaciones Estéticas por el centenario de su nacimiento. Estos homenajes consistieron en: "Velada Solemne" en la que se canceló un sello postal con la efigie del Dr. Manuel Toussaint que se llevó a cabo el 26 de mayo en la ex-Capilla del Palacio de Minería.

Coloquio Internacional Extraordinario Manuel Toussaint. Su proyección en la historia del arte mexicano, los días 30 y 31 de julio y del 1 al 3 de agosto, en La Trinidad, Tlaxcala, contando con el patrocinio del gobierno del Estado.

Con el Museo Nacional de Arte la exposición Obras maestras del Arte Colonial. Homenaje a Manuel Toussaint (de agosto a octubre). Gran parte de esta muestra se presentó del 15 de noviembre de 1990 al 15 de enero de 1991) en la ciudad de Monterrey.

El 14 de septiembre se develó la placa que informa que el Archivo Fotográfico del Instituto de Investigaciones Estéticas, lleva el nombre de su fundador Manuel Toussaint.

Se organizó la exposición fotográfica Paseos Coloniales. Homenaje a Manuel Toussaint, del 12 al 23 de noviembre en la Casa Universitaria del Libro. Dentro del marco de esta exposición se llevó a cabo la Semana del Libro de Arte del Instituto de Investigaciones Estéticas.

En colaboración con el Instituto Mexiquense de Cultura y de igual manera conjuntamente con el Patrimonio Cultural de Occidente, se realizaron mesas redondas y conferencias en homenaje a José María Velasco en la Casa de Cultura de Toluca, Estado de México (4 de julio) y en la ciudad de Guadalajara ( 23 y 24 de julio).

Contando con el inapreciable apoyo del Instituto Veracruzano de Cultura, dirigido por la doctora Ida Rodríguez Prampolini, investigadora emérita de nuestro Instituto, y el gobierno del estado de Veracruz, se organizó el xIV Coloquio Internacional, titulado: Encuentros y desencuentros en las artes, que tuvo lugar del 24 al 28 de septiembre, en la sede del IVEC. 
En colaboración con el gobierno del estado de Guerrero, la Federación Mexicana de Universitarias y la Facultad de Arquitectura, se llevó a cabo el Seminario Santa Prisca de Taxco. Una revisión histórica y estética (12 y 13 de octubre) en Taxco Guerrero.

En el marco del proyecto especial Música, Matemáticas y Computación, realizó el Seminario Año 2000. Alternativas teóricas, tecnológicas y composicionales como homenaje al precursor de la música por computadora Conlon Nancarrow, con la colaboración del Instituto de Investigaciones en Matemáticas Aplicadas y en Sistemas, Dirección General de Asuntos del Personal Académico y Escuela Nacional de Música, así como de los Institutos Francés de América Latina y Goethe del 22 al 24 de octubre.

También dictó conferencias en esos actos académicos y en otras de la Universidad, algunos de los cuales se enlistan a continuación:

Dentro del homenaje organizado por la Coordinación de Difusión Cultural en Honor de los maestros transterrados la conferencia "Dos maestros de la Escuela Nacional de Artes Plásticas".

"José María Velasco fortuna crítica" en el homenaje al pintor en las ciudades de Toluca y Guadalajara.

"Manuel Toussaint una devoción barroca" en el homenaje a Manuel Toussaint en el Centro Cultural Alfa de Monterrey, N.L.

Publicó el libro El Pintor Nicolás Moreno, México, Banco Nacional de Obras y Servicios Públicos, Fondo Editorial de la Plástica Mexicana, 1990.

El maestro José Rogelio Ruiz Gomar Campos participó en el II Congreso Regional de Historia Comparada, organizado por la Universidad Autónoma de Ciudad Juárez, los días 27 al 30 de marzo, con la ponencia "La pintura virreinal en Chihuahua".

Además de sus cursos en la Facultad de Filosofía y Letras de la vNAM, impartió tres cursillos. Uno sobre "Pintura manierista en la Nueva España" y otro sobre "Pintura barroca novohispana" organizados por el Museo Nacional de las Intervenciones, INAH, los meses de febrero y mayo. Y un tercero, intitulado "Pintura en la Nueva España", dentro del Diplomado en Historia del Arte que ofreció la Universidad Iberoamericana en el mes de octubre.

A invitación de la Casa de Cultura de San Luis Potosí, dictó una conferencia sobre "La pintura en la Nueva España" en el mes de agosto. 
Dentro del programa "Paseos Culturales del INAH" llevó siete visitas guiadas, entre las que destacan las que hizo con el propósito de conocer la pintura virreinal que se conserva en las ciudades de Tlaxcala, Querétaro, Zacatecas, San Miguel Allende y Guanajuato.

Elaboró seis fichas para el catálogo de la gran exposición de Arte Mexicano en el Metroplitan de Nueva York, y cinco más para el catálogo de la exposición en homenaje a don Manuel Toussaint verificado en el Museo Nacional de Arte (INBA).

El maestro Julio Estrada quedó inscrito en estudios de Doctorado en la Université de Strasbourg II, Francia. Becas del gobierno francés de Alto Nivel Científico y Técnico y de la Dirección General de Asuntos del Personal Académico, UNAM, para la realización de sus estudios. Ingresa a la Sociedad Española de Musicología. En la Sociedad Astaldi, Roma y en el Seminario de Composizione de la Civica Scuola di Musica Milano, realiza dos cursos basados sobre su teoría del Espectro Interválico. Es nombrado Responsable del Proyecto de Investigación Música, Matemáticas y Computación (DGAPA, IIE e IMMAS, UNAM). Organiza en el IIE el Seminario Internacional año 2000; participantes: Dr. Roger Dannenberg, Julio Estrada, Dr. Jurgen Hocker, Jack Jarret, Candido Lima, Francois Bernard Mache, etc. Como Director de Música, Sociedad de Interpretación, Investigación, Creación (MusSIIC), organiza diversas actividades en colaboración con dependencias universitarias: serie de siete conciertos y una exposición "Conlon Nancarrow: 50 años en México" (DGAPA, IIE); cuatro conferencias magistrales de músicos italianos en México: Aldo Brizzi, Stefano Scodanibbio, Federico Mondelci y Giancarlo Schiaffini (ENM); un curso sobre Computación y Música, con el Dr. Jack Jarret y el Dr. Roger Dannenberg (IIE, IIMAS). También, un "Homenaje a Rulfo", 4o. aniversario de su muerte, librería Bajo el Volcán, Cuernavaca, Morelos. Con MúsSIIC promueve el estreno en México de obras de Baca, Iannis Xenakis, Ligeti, Rzewski y Nancarrow, entre otros.

Responsable de la organización y catalogación del archivo musical de Jacobo Kostakowsky, donado al IIE por la familia Kostakowsky.

Participación en congresos: "Silencio y rumor de lo propio", (Coloquio Encuentros y desencuentros, IIE), "La música en la UNAM: retrospectiva y perspectivas" (Congreso Universitario) y "Los sistemas 
Espectro Interválico y Eua'Oolin: desarrollos recientes" (Seminario año 2000, DGAPA, IIE, IIMAS). Publica nueve artículos de divulgación (Los Universitarios, Sábado y La Jornada). Publica el libro El sonido en Rulfo, con 17 fotografías inéditas de Rulfo, coedición del Instituto de Investigaciones Estéticas y la Coordinación de Difusión Cultural, UNAM. Enero 6, 1990. Produce dos programas para TUUNAM en colaboración con Oscar Menéndez: El sonido en Juan Rulfo y "Dos cuentos de El Llano en llamas, de Juan Rulfo". En su obra de compositor asociada a sus investigaciones, concluye Ishini'ioni, cuarteto de cuerdas; Yuunohuio'ce, violín; Yuunohui'ome, viola y Nahui Yuunohui, publicadas por Editions Salabert, París. Irvine Arditti graba en Francia en disco compacto el Canto oculto, violín.

Aparte de las actividades propias del cargo que ocupa de Director General de Radio UNAM el investigador Alberto Dallal asumió la coordinación de los Anales del Instituto de Investigaciones Estéticas. Continuó la catalogación del Fondo de Fotografías de Danza (cubrió datos para 1550 de un total de 2500). Continuó la redacción del libro La danza en México. Tercera parte, que presentará a consideración del Comité Editorial del Instituto próximamente. Terminó la revisión, corrección y actualización de originales para la tercera edición de La danza contra la muerte. Fue nombrado miembro de la Comisión de Artes y Letras del Fondo del Consejo Nacional para la Cultura y las Artes. Asimismo, elaboró 30 fichas para el Diccionario de Música Española e Hispanoamericana. Dirige una tesis de licenciatura sobre Elías Nandino en la Facultad de Ciencias Políticas y Sociales, en donde inició su curso "Géneros periodísticos interpretativos" (12 de noviembre). Dictó las siguientes conferencias: "Extensión y difusión de la cultura", CCH-Azcapotzalco (24 de enero), "Lectura, escritura y cultura", Preparatoria "Antonio Caso" (2 de abrili), "Géneros periodísticos en la radio", Universidad Autónoma del Estado de Morelos (8 de septiembre), "El arte actual, la ciencia y la tecnología", Escuela Popular de Bellas Artes, Morelia (27 de noviembre), "Periodismo cultural en los medios (radio)", Casa de la Cultura de Oaxaca (1 de diciembre). Impartió el curso "Radio y televisión cultural" en Tuxtla Gutiérrez (6 de noviembre). Participó como ponente en la presentación de los Cuadernos del Centro de Relaciones internacionales, UNAM ( 3 de mayo) y participó como ponente en el XIV 
Coloquio Internacional del Instituto de Investigaciones Estéticas en Veracruz. Tema de la ponencia: "Originalidad, asimilación y capacidad de síntesis en las técnicas dancísticas. El caso del Ballet Nacional de México" (24 de septiembre). Durante 1990 publicó 23 artículos de divulgación y los siguientes de investigación:

"Anna Pávlova en México", Anales del IIE, UNAM, núm. 60, 1989, pp. 163-178.

"Tradición y cambio", El Correo Escénico, México, núm. 1, febrero de 1990.

"José Limón, El Correo Escénico, núm. 2, marzo de 1990.

Escribió la presentación del libro de Gloria Contreras: Ballet paso a paso, Difusión Cultural, UNAM, 1990.

Publicó los siguientes libros:

Lenguajes periodústicos, Instituto de Investigaciones Estéticas, UNAM, 1990. $110 \mathrm{pp}$.

El aura del cuerpo, Instituto de Investigaciones Estéticas, UNAM, 1990,160 pp.

La mujer en la danza, Panorama Editorial, 1990, $182 \mathrm{pp}$.

La maestra Louise Noelle Mereles asistió al Congreso Binacional "Arquitectura de la Frontera, Mexicali, B.C., en febrero, con la ponencia "Arquitectura Regional. Algunos ejemplos", al primer simposio internacional "Luis Barragán", México, D.F., en marzo, con la ponencia "Presencia de Artistas Europeos en la obra de Luis Barragán", al taller de la Academia Internacional de Arquitectura, Boukhara, Uzbekistan, URSS, en abril, con las ponencias "La obra maestra de Luis Barragán" y "Los problemas de la Arquitectura Regional", y al xVII Congreso Mundial de la Unión Internacional de Arquitectos, Montreal, Canadá, en mayo, con las ponencias "Los escritos de Arquitectura más relevantes de los últimos tres años" y "Tendencias actuales de la Arquitectura Mexicana". Fungió como miembro del Comité Organizador de los Coloquios del Comité Mexicano de Historia del Arte, "Arte y Coersión", México, D.F., y del Instituto de Investigaciones Estéticas, "Encuentro y desencuentro en las artes", Veracruz, Ver.

Dictó diversas conferencias y publicó artículos en revistas especializadas. Continuó con su labor como Cronista de la Academia Nacional de Arquitectura, y como Secretaria del Comité Mexicano de Historia 
del Arte. Fue electa Consejera Representante del Personal Académico del Instituto de Investigaciones Estéticas, en junio. Fungió como jurado del concurso de estudiantes del xvi Congreso de la ULA, Montreal, Canadá, en mayo, y de la Primera Bienal de Arquitectura Mexicana, Monterrey, N.L., en julio. 